\title{
Application of Microscrew Implant Anchorage in Orthodontics
}

\author{
Hiuching Wong*
}

China Medical University, Shenyang 110000, Hong Kong, China. E-mail: 417181345@qq.com

\begin{abstract}
Objective: To study the value of microscrew implant anchorage in orthodontic treatment. Methods: A total of 80 cases received orthodontic treatment in recent two years were selected and divided into experimental group and control group based on their received orthodontic measures, each group contains 40 cases. The control group was treated with general orthodontic treatment plan, while the study group received microscrew implant anchorage for the treatment. The clinical conditions of the two groups were counted and observed. Results: After different treatments, the effect of the study group was significantly better than that of the control group. All the indicators (including the improvement of molar displacement, incisor inclination angle and incisor convex distance) were available. In addition, there are obvious differences between the two groups in the occurrence of adverse reactions including inflammatory reaction, soft tissue edema and discomfort. Conclusion: In oral clinic, microscrew implant anchorage can achieve ideal curative effect for those who need orthodontics. Besides, its safety is relatively high, which is worth popularizing widely.
\end{abstract}

Keywords: Orthodontics; Microscrew Implant Anchorage; Safety

\section{Introduction}

In stomatological clinic, orthodontic intervention is also called orthodontics, a common treatment method adopted by the department of stomatology. Good orthodontic intervention can improve recessive diseases to a great extent, and can also effectively clean teeth and beautify faces ${ }^{[1]}$. In traditional orthodontic treatment, most use external arch or transverse palatal bar. There are many reports of adverse reactions in clinic. In recent years, with the development and progress of stomatology, microscrew implant anchorage (hereinafter referred to as microscrew) has been widely recognized, and its application prospect is also ideal ${ }^{[2]}$. In this research, orthodontic effect of microscrew implant anchorage is studied in the following contents.

\section{Data and methods}

\subsection{General information}

A total of 80 cases who received orthodontics in recent two years (December 2018 to May 2020) were selected. Due to symptoms such as dental caries, swollen gums, loose teeth, all of the cases went to see doctors. Participations in this research have ruled out the special circumstances, such as unconsciousness, unwillingness to cooperate with the study, and major organic diseases. Experimental group and control group were divided according to the orthodontic treatment measures. Each group contains 40 cases: the study group covers 25 males and 15 females, aged from 22 to 39 years old; while the control group included 24 males and 16 females, aged from 23 to 40 . There is no significant difference in general data. The research conforms to ethical standards and it can be carried out.

\subsection{Methods}

General anchorage treatment was given to the control group Microscrews were given about 8 weeks before the tooth extraction gap was closed. Firstly, lidocaine

\footnotetext{
Copyright (C) 2021 Hiuching Wong

doi: 10.18686/aem.v10i1.188

This is an open-access article distributed under the terms of the Creative Commons Attribution Non-Commercial License

(http://creativecommons.org/licenses/by-nc/4.0/), which permits unrestricted non-commercial use, distribution, and reproduction in any medium, provided the original work is properly cited.
} 
(2\%) was taken for local anesthesia, and then a longitudinal gingival margin between the roots of the maxillary first molars and second canines. A micro-screw implant was then placed in the left and right sides respectively. After implantation, a load pressure of about 5g-200g was applied between the implant nail, the maxillary and mandibular arch wire traction hooks at the time points of 2 weeks, 4 weeks and 8 weeks. Patients were instructed to make regular follow-up visits. At the same time, repeated stress was applied with reference to the closure of the patient's tooth gap until the tooth extraction gap was completely closed.

The experimental group received microscrew implant anchorage, which covered cleaning the periodontal tissues of patients. Cavities were filled in time for treatment if they were found. If there were other oral diseases, they needed to be timely treated to avoid burying orthodontic risks. In such methods, it is necessary to select straight wire arch metal bracket orthosis, to adjust the upper teeth and lower teeth of the case to be flush, to adduce the whole teeth by sliding stainless steel wire, and to close the extraction gap at the same time. During the treatment, patients should use anchorage devices correctly, and strengthen traction with extraoral arch and traction technique to ensure traction intervention for about 10 hours to 12 hours every day.

In addition to the treatment above, adjuvant treatment and nursing measures received by the two groups were completely the same.

\subsection{Clinical observation indicators}

Referring to the head X-ray lateral scan films of the two group's cases, the clinical prognosis was compared and observed to count and observe the clinical efficacy. In addition, according to the investigation of patients' clinical manifestations and complaints, the adverse reactions after receiving intervention were determined. Specifically, clinical observation indicators mainly cover the following two aspects.

The first, clinical indicators include the improvement of molar displacement, incisor inclination angle and incisor convex distance.

The second, adverse reactions include inflammatory reaction, soft tissue edema, discomfort (chief complaint).

\subsection{Statistical methods}

Referring to the data requirements put forward in the observation index, statistical software is selected to process the data. The counting data involving probability and percentage are expressed by (probability/\%) and confirmed by the line. The measurement data involving variables are expressed by (quantitative variables) and tested. The statistical results show that the $P$ value is below 0.05 , which means the difference is significant.

\section{Results}

Clinical efficacy of 2.180 cases refers to Table 1. Adverse reactions of 2.280 cases refer to Table 2 .

Table 1. Comparative analysis of clinical efficacy of the 80 cases

\begin{tabular}{|l|l|l|l|}
\hline Group & $\begin{array}{l}\text { Molar displacement } \\
\text { (unit: } \mathbf{~ m m})\end{array}$ & $\begin{array}{l}\text { Inclination angle of incisors } \\
\text { decreases (unit: }{ }^{\circ} \text { ) }\end{array}$ & $\begin{array}{l}\text { Decreased incisor pitch } \\
\text { (unit: } \mathbf{~ m m} \text { ) }\end{array}$ \\
\hline Experimental group $(\mathrm{n}=40)$ & $3.24 \pm 0.19$ & $29.31 \pm 7.02$ & $4.31 \pm 1.65$ \\
\hline Control group $(\mathrm{n}=40)$ & $5.23 \pm 0.69$ & $13.24 \pm 4.57$ & $2.74 \pm 0.84$ \\
\hline T value & 17.586 & 12.133 & 5.363 \\
\hline P value & & & \\
\hline
\end{tabular}

Table 2. Comparative analysis of adverse reactions in the 80 cases

\begin{tabular}{|l|l|l|l|}
\hline Group & Inflammatory lesions & Significant discomfort & Soft tissue edema \\
\hline Experimental group $(\mathrm{n}=40)$ & $1(2.50 \%)$ & $1(2.50 \%)$ & 0 \\
\hline Control group $(\mathrm{n}=40)$ & $4(10.00 \%)$ & $3(7.50 \%)$ & $1(2.50 \%)$ \\
\hline Chi-square value & 4.114 & & \\
\hline P value & 0.043 & \\
\hline
\end{tabular}




\section{Discussion}

Most common cases requiring orthodontics include mesioclusion, convexity, malposition of teeth, etc., in medical clinic. Most of the deformities are caused by patients' daily diet, breathing style, congenital heredity and other factors. If treatment is not given in time, there will be great hidden dangers that will lead to loose teeth and slurred speech ${ }^{[3]}$. In addition, it requires more difficult techniques for oral deformity to clean oral cavity, which could increase the risk of alveolar bone and soft tissue damages, and then affects the normal chewing function ${ }^{[4]}$. Therefore, increasingly clinical medical workers and researchers pay attention to how to apply orthodontic intervention correctly in clinic.

In this study, the positive effects of microscrew implant anchorage on orthodontic patients were analyzed. Compared with the control group, patients in the experimental group showed obvious advantages in both clinical efficacy and the probability of adverse reactions. The latter was significantly lower in the experimental group. This achievement is reliable, which has also been confirmed in Huang Shanxia's ${ }^{[5]}$ and Sun Yuchao's works ${ }^{[6]}$.

The overall control quality of anchorage is generally the key in orthodontics. It is also a key prerequisite to ensure the successful completion of treatment. On the whole, extraoral arch and maxillofacial traction are traditional orthodontic anchorage control methods, and the treatment effect is ideal. However, due to its long time-consuming cycle, complicated practical operation, unstable reduction effect, high probability of patients complaining of discomfort and other problems, its clinical application is controversial. Besides, this method demands a high degree of cooperation from patients, which directly determines whether the treatment effect meets the clinical needs. During the recovery of front teeth, there is a close relationship between the anchorage teeth and facial bones, jaws and muscles, which is very easy to induce serious anchorage teeth loosening prob$\operatorname{lems}^{[7]}$.

The stability and resistance of microscrew implant anchorage are both ideal and clinic. This method can adjust the facial morphology of patients, to a great extent. In addition, patients will experience less foreign feelings and can adapt to it after having been placed for two weeks. Patients do not need to cooperate actively during operation. When the anchorage is implemented, it does not need the guidance of bone bricks. With the help of the rotation of threaded nails, it can smoothly enter the bone and be fixed with good mechanical strength, greatly reducing time for osseointegration ${ }^{[8]}$. During the treatment, new bones can provide good support and overall stability for implants. Indications of micro-screw anchorage implants are extensive, which needs to be mastered in clinical practice. Besides, attentions need to be paid to contraindications, such as anchorage molar advancement and periodontitis ${ }^{[9]}$. When choosing the position of implant, doctors should try to make a comprehensive evaluation with reference to the patient's age, anchorage demand, bone cortex thickness and bone morphology in the implantation area, in order to improve clinical adaptability ${ }^{[10]}$.

\section{Conclusion}

In summary, microscrew implant anchorage can achieve ideal curative effect and relatively high safety for patients who need orthodontic treatment. It is worthy of in-depth clinical practice and extensive theoretical research in oral clinic to bring convenience to more patients who need orthodontic treatment.

\section{References}

1. Guo W. Application effect of micro-screw implant anchorage in clinical orthodontic treatment (in Chinese). World Latest Medicine Information 2019; 19(81): 120, 126.

2. Zhu Y. Clinical application of microscrew implant anchorage in orthodontics. Systems Medicine 2019; 4(14): 124-126.

3. Zhong S, Liu H, Zhong S, et al. Clinical application of microscrew implant anchorage nail in orthodontic treatment. Chinese Medical Innovation of China 2019; 16(32): 5-9.

4. Wang H, Qiao Y. Research progress of microscrew implant anchorage in orthodontic application. Chinese Journal of Practical Medicine 2019; 46(16): 123-126.

5. Huang S. Clinical observation of micro-screw anchorage implant in orthodontic treatment (in Chines). Electronic Journal of General Stomatology 2019; 6(33): 47, 55.

6. Sun Y. Comparison of orthodontic value between conventional orthodontics and micro-screw implant anchorage in orthodontics (in Chinese). Jian Kang Zhi You 2020; 1(4): 90-91.

7. Yuan Y. Comparison of therapeutic effects between micro-screw anchorage implant and traditional strong anchorage orthodontic method in orthodontics. China Medical Device Information 2020; 26(5): 
134-135.

8. He M, Yin Z, Guo L. Comparative study on orthodontic effect of micro-screw anchorage implant and extraoral arch anchorage (in Chinese). Electronic Journal of General Stomatology 2019; 6(20): 57.

9. Fang L, Li L, Pan D. Value analysis of conventional extraoral arch anchorage and micro-screw anchor- age implants in orthodontics (in Chinese). Electronic Journal of General Stomatology 2019; 6(13): $56-57$.

10. Xiang Y. Clinical application value of micro-screw implant anchorage in orthodontics (in Chinese). Health Guide 2019; 1(23): 72. 